\title{
Exploring Multimodal Robotic Interaction through Storytelling for Aphasics
}

\author{
Omar Mubin \\ Dept. of Industrial Design \\ Eindhoven University of Technology \\ Den Dolech 2, 5600 MB, Eindhoven \\ The Netherlands \\ $+31402473842$ \\ o.mubin@tue.nl
}

\author{
Abdullah Al Mahmud \\ Dept. of Industrial Design \\ Eindhoven University of Technology \\ Den Dolech 2, 5600 MB, Eindhoven \\ The Netherlands \\ $+31402472147$ \\ a.al-mahmud@tue.nl
}

\begin{abstract}
In this poster, we propose the design of a multimodal robotic interaction mechanism that is intended to be used by Aphasics for storytelling. Through limited physical interaction, mild to moderate aphasic people can interact with a robot that may help them to be more active in their day to day activities. In our proposed design Aphasics interact with the iCat robot which recognizes and understands the gestures of Aphasics hereby enabling both of them to participate in a one to one interaction. This interaction helps Aphasics to share and reinforce their experience of storytelling and consequently helps them to reengage in their post-stroke life.
\end{abstract}

\section{Categories and Subject Descriptors}

H.5.2. [Information interfaces and presentation] (e.g., HCI): User Interfaces - User-centered design

\section{General Terms}

Design, Experimentation, Human Factors.

\section{Keywords}

Human-Robot interaction, Aphasia, Multimodal interaction, Gestures, ACC devices, Sharing experience, Storytelling.

\section{INTRODUCTION}

Aphasia is a communication disorder caused mainly due to brain injury that affects the ability of Aphasics to speak and articulate their thoughts [6]. This leads to a sense of deprivation, social isolation and lack of confidence. Our aim is to design a multimodal interaction mechanism primarily by adopting gestures that can assist Aphasics in conveying their stories to a robot and consequently to their family members, caregivers and other social partners. We claim that by enabling them to interact with a social and embodied agent (such as a robot) that they can collaborate and communicate with, there exists a possibility of them overcoming their lack of confidence and effectively directing their daily stories to anyone that they wish. Consequently, sharing experiences through stories will help aphasics in eliminating their social isolation.

(c) The Author 2008

Published by the British Computer Society
Aphasics have a strong bonding with their partner (husband/wife), to whom they can explain what they mean by exhibiting gestures or otherwise. Therein exists potential in a robot taking the role of a surrogate partner when the real partner of the aphasic is not nearby or not at home. The only communication devices for aphasics are of the Alternative and Augmentative Communication (AAC) [7] types which help Aphasics for need based interaction but they do not interact as a communication partner or helper. That is a limiting factor and investigation is required to locate opportunities for more meaningful ways of communication for Aphasics. Robots have been used to provide cognitive assistance to patients suffering from Dementia, Alzheimer and other related disorders [2, 4]. By interacting with robots, patients can feel a sense of companionship, motivation and removal of isolation.

As far as we are aware of, within the domain of assistive robotics there has been no attempt to enhance the social and psychological well being of aphasics in general. In this sense, our suggested design would be novel in itself. Since we are aware of the exact storytelling requirements of aphasic patients, we can design an assistive interaction mechanism accordingly. There are a couple of related examples of storytelling robots [e.g., 3] but they were not created for our target group.

We aim to evaluate if a robot can be effective in enhancing the social well being of aphasics. As stated prior, off the shelf traditional AAC (Alternative and Augmentative Communication) devices (e.g., TouchSpeak [5] ) fulfill a need based requirement but do not support two-way communication. Therefore our design will attempt to enhance two way communications between aphasics and robot.

\section{USER STUDY AND SCENARIO}

To gain an understanding of the user group we visited several Aphasia rehabilitation centers and interviewed speech therapists and caregivers. We did an ethnographical user study, in which we observed how aphasics ( $\mathrm{N}=8$, spread across two locations, each group for one hour) would interact using gestures with peers, speech therapists and caregivers. It was observed that Aphasics used gestures and pointing as the primary form of communication. They express emotions, they cry, laugh the same way as normal human beings, only without expressing words.

A Robot can be used to train Aphasics especially for therapy purpose (e.g. dialogue systems and encouraging them to express stories). Story creation can be possible with the help of robot. Gestures can be employed as the mode of interaction since aphasics not only understand gestures but can also use them. A Robot can be a communication partner or can be used as a helper to carry out daily activities, such as preparing coffee in the kitchen, vacuum cleaning the house, etc. 
The following scenario illustrates how the proposed interaction mechanism can help Aphasics. This scenario was acquired from the results of our user study phase.

Peter is an Aphasic person. He is heavily dependent on his wife Annie to carry out daily activities. Annie goes for her job everyday. Therefore, she cannot be with Peter during her office time. Fortunately Peter has a companion robot which helps him to remove is isolation and keeps him engaged while Annie is away from home. The robot and Peter understand each others and the robot also helps him to carry out his daily activities. Peter can also build/express his story with the help of the robot. Peter doesn't feel lonely anymore. This has really changed Peter's life. Now he is more confident and psychologically getting well day by day.

\section{CONCEPT DESIGN}

It is clear that since aphasics have a limited capacity to indulge in verbal communication, the interaction should be multimodal (e.g. gesture, annotation, and limited speech). To express a particular concept from the real world such as drinking coffee the aphasic can carry out a specific gestural command. Herein exist two possibilities: either the robot can recognize gestures of the aphasics (sign language or the basic action/activity, see Figure 2) or it can recognize pointing/pressing of particular icons. The icons can be placed on a digital tabletop for example (or any digital screen for that matter). The aphasic can then indicate a specific component of his/her story by activating that icon, in this case the act of drinking a coffee. In both cases, the robot would initiate the conversation by affirming the recognition process it has carried out.

We carry out our implementation by using the iCat robot [1]. The iCat is a research platform to study human robot interaction that can enable us to carry out our interaction design in terms of speech recognition/generation and gesture recognition. The robot is $38 \mathrm{~cm}$ tall and is equipped with 13 servos that control different parts of the face, such as the eyebrows, eyes, eyelids, mouth, and head position. With this setup, iCat can generate many different facial expressions, such as happiness, surprise, anger, or sadness. There is a built in camera inside the iCat that will be used for gesture recognition. The architecture of the iCat is such that it allows researchers to augment their own software modules on top of the existing framework. Given below (Figure 1) is a chart which illustrates the sequence flow of the interaction mechanism.

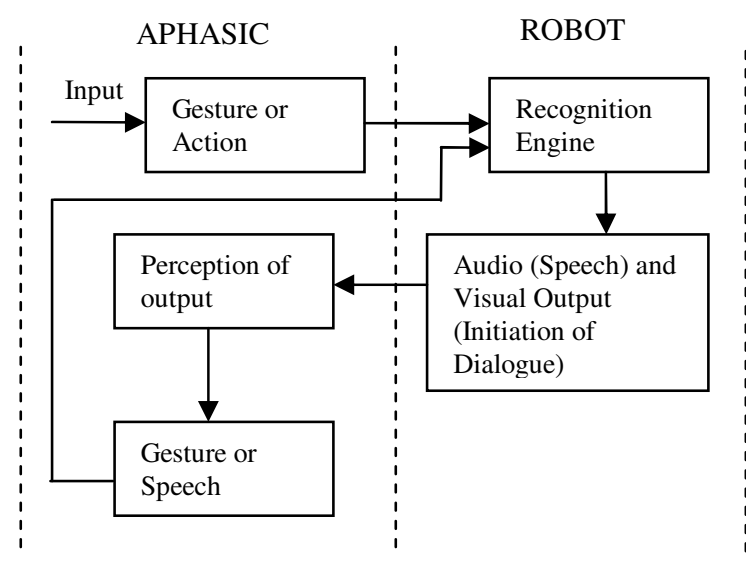

Figure 1. Interaction mechanism
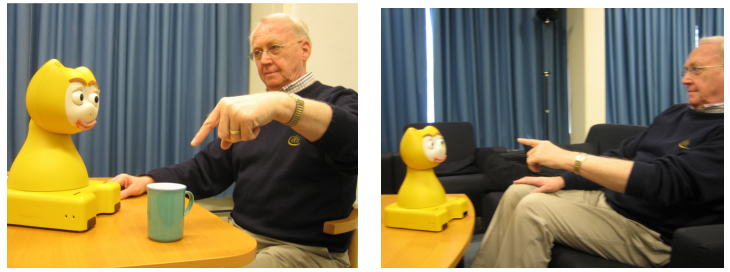

Figure 2. Gesturing with iCat

\section{EVALUATION PLAN}

Our plan is to first carry out a pilot study with proxies such as caregivers and partners of Aphasics to test the working of the system, in terms of the robustness of the recognition. By doing a pilot evaluation we can also determine if our proposed interaction mechanism is realistic and acceptable. In the next phase we wish to evaluate our interaction mechanism with mild aphasics in local Aphasia centers, whose verbal expression condition is not that severe. Finally, we would conduct a longitudinal evaluation study, in which the Aphasics will be provided an opportunity to interact with the iCat for a number of weeks.

\section{CONCLUSION}

There are two add on effects of designing such an interaction mechanism for aphasics. Firstly, our primary goal of providing them with a storytelling tool to share experiences is achieved, which will enable the aphasics to overcome social isolation and achieve psychological well being. Secondly, we can evaluate any therapeutic consequences of employing the use of such an interaction mechanism.

\section{ACKNOWLEDGMENTS}

We are grateful to Reijndam Rehabilitation Centrum, Rotterdam for their cooperation and guidance.

\section{REFERENCES}

[1] iCat.http://www.research.philips.com/technologies/syst_so ftw/robotics/

[2] Matarić, M.J., Jon Eriksson, David J. Feil-Seifer, and Carolee J. Winstein. "Socially Assistive Robotics for PostStroke Rehabilitation". In Journal of NeuroEngineering and Rehabilitation, 4(5), Feb 2007

[3] Shih, Chao-Fen., Chang, Chih-Wei., Chen, Gwo-Dong., Robot as a Storytelling Partner in the English Classroom Preliminary Discussion. In Seventh IEEE International Conference on Advanced Learning Technologies, 2007. ICALT 2007,18-20 July 2007 Page(s):678 - 682

[4] Tapus, A., Fasola, J., and Matarić, M.J., "Cognitive Assistance and Physical Therapy for Dementia Patients". In ICRA 2008, Workshop on Social Interaction with Intelligent Indoor Robots, May 2008.

[5] TouchSpeak. http://www.touchspeak.co.uk/

[6] The aphasia institute: What is aphasia? Available at http://www.aphasia.ca/about/whatis.html//encyclopedia

[7] van de Sandt-Koenderman, M., Wiegers, J. and Hardy, P. A Computerised Communication Aid for People with Aphasia. Disability and Rehabilitation, 27(9), 529-533, 2005.

[8] van de Sandt-Koenderman, High-tech ACC and aphasia: widening horizons? Aphasiology, 18(3), 245-263, 2004. 